\title{
El léxico español del vuelo: un proyecto lexicográfico para el dominio del transporte aéreo
}

\author{
Lorena M. A. de- Matteis ${ }^{1}$ \\ Universidad Nacional del Sur, Argentina
}

\begin{abstract}
The lexicon of air transport has received the influence of terms from other modalities of locomotion, of sciences such as meteorology and ornithology and it has also incorporated loan words from different languages. Although it has been the object of lexicological study in different languages, there are few studies that address the appropriation of this lexicon by non-specialists and no dictionary exists that accounts for the non-technical uses of such lexical units. This article presents the lexicographic decisions adopted to design El léxico español del vuelo, a proposal that attempts to record the daily use of Spanish aeronautical words, giving an account of their diatopic, diachronic and diaphasic variation. Specifically, it describes both the selection of materials and the conformation of the lexical database as well as the text macrostructure, the criteria that operated in the lemmas selection and ordering and, finally, the microstructure of the lexicographic articles.
\end{abstract}

Keywords: lexicography, specialized lexicons, daily speech, Spanish

\section{RESUMEN}

El léxico del transporte aéreo se ha nutrido con el trasvase de términos de otras modalidades de locomoción, de ciencias como la meteorología o la ornitología y ha incorporado préstamos de distintas lenguas. Si bien ha sido objeto de estudio lexicológico en distintas lenguas, existen escasos estudios que aborden la apropiación de este léxico por los no especialistas y ningún diccionario de español que dé cuenta de los usos no técnicos de tales unidades léxicas. Este artículo presenta las decisiones lexicográficas adoptadas para diseñar El léxico español del vuelo, propuesta que intenta registrar el uso cotidiano de voces aeronáuticas, dando cuenta de su variación diatópica, diacrónica y diafásica. En términos específicos, se describe tanto la selección de materiales y la conformación de la

Correspondencia: Departamento de Humanidades. 12 de octubre 1198-6 $\mathrm{P}^{\circ}$-Gab. $18 \mathrm{~A}$. B8000CTX -Bahía Blanca. Pcia. De Buenos Aires. República de Argentina.

Email: Iorelei.dematteis@gmail.com 
El léxico español del vuelo: un proyecto lexicográfico para el dominio del transporte aéreo

base de datos léxica como la macroestructura del texto, los criterios que operaron en la selección y ordenamiento de los lemas y, por último, la microestructura de los artículos del diccionario.

Palabras clave: lexicografía, léxicos especializados, habla cotidiana, español

\section{Introducción}

Las lenguas de especialidad pueden definirse como "subconjuntos de recursos específicos, lingüísticos y no lingüísticos, discursivos y gramaticales, que se utilizan en situaciones consideradas especializadas por sus condiciones comunicativas (Cabrè y Gómez de Enterría, 2006: 12). Como variedades "subsidiarias de la lengua común" (Gómez de Enterría Sánchez, 2009: 21), comparten con ella tanto la sintaxis como los procedimientos para la formación del léxico, pero se diferencian porque presentan características propias en los distintos niveles de análisis. Así, por ejemplo, el lenguaje de la aviación registra preferencias por la acronimia como recurso de creación léxica (por caso, radar) y ciertas interacciones se caracterizan en el nivel pragmático por una muy baja personalización del discurso y por la formalización de las prácticas comunicativas bajo la forma de "procedimientos estandarizados", con una fraseología radiotelefónica de sintaxis elíptica.

Pero, sobre todo, las lenguas de especialidad se distinguen por el nivel léxico, cuyo estudio adquiere un gran interés. Aunque estas variedades comparten con la lengua común parte del vocabulario básico, entendiendo esta denominación en sentido amplio, como "el referido a ciertas cosas y situaciones o clases de cosas y situaciones que son recurrentes y para las que todos los seres humanos, sean cuales sean su cultura y su medio, disponen de términos que los designen" (Fontanella de Weinberg, 1995: 167), poseen también una terminología propia que resulta opaca a los hablantes que no forman parte del grupo profesional y que, por necesidades pragmáticas de los campos disciplinares, tiende hacia la univocidad de sus términos.

A la inversa, algunas expresiones surgidas como terminología propia de una especialidad penetran luego en la lengua general, por caso la voz aviación, que designaba en sus orígenes franceses a la posibilidad de 'volar a la manera de las aves', esto es, con una dirección intencional. Como término, esta unidad puede contraponerse en el ámbito especializado a aerostación, en tanto se vale de vehículos fundados en el principio de lo "más pesado que el aire" mientras que la aerostación se basa en el principio opuesto. Esta oposición suele ser desconocida por 
el hablante lego, quien emplea aviación con sentido general ${ }^{2}$. Señala Gómez de Enterría Sánchez sobre el proceso de penetración de estos términos en la lengua general:

Las lenguas de especialidad son, sin lugar a dudas, patrimonio de los especialistas que las emplean para llevar a cabo la comunicación científica, tecnológica y profesional. Sin embargo, hoy observamos que, en numerosas ocasiones, pueden sobrepasar los ámbitos de especialidad y llegar hasta la lengua común. Este fenómeno se produce gracias a la intensa labor que ejercen continuamente los medios de comunicación. (...) Lo que nos lleva a afirmar que muchas veces es difícil marcar los límites que existen entre lengua común y lengua de especialidad, especialmente en sectores como el de los productos financieros, la cosmética, la nutrición, la electrónica, etc. (...) (2009: 22-23).

Como en tales sectores, para el caso del transporte también influyen los medios de comunicación en el proceso de penetración de los términos en la lengua general. A esto se suma el hecho de que los hablantes son clientes o usuarios del transporte aéreo y, por tanto, hacen uso de parte del léxico especializado, aunque empleándolo sin las características de precisión que lo caracterizan en el ámbito de uso original.

Los léxicos de especialidad, por tanto, constituyen un área de particular desarrollo dentro de los estudios orientados hacia la enseñanza de lenguas y la traducción. La práctica lexicográfica más frecuente es la preparación de diccionarios terminológicos que pueden ser mono-, bi- o plurilingües, en función del público al que están destinados (por ejemplo, estudiantes o profesionales, traductores especializados, docentes o periodistas). Algunas de estas voces especializadas pueden aparecer también en diccionarios de lengua general, que las incluyen con una marcación temática que señala la disciplina a la que pertenecen y proporciona información pragmática sobre su empleo (Estopá, 1998). Pero los diccionarios de lengua general pueden incluir un número limitado de estas expresiones y son muy pocos los que, sin ser terminológicos, se orientan a registrar las voces de origen especializado empleadas en el uso cotidiano sin afán de precisión semántica ni conceptual. Un ejemplo son los 23 diccionarios que integran la colección "La Academia y la lengua del pueblo", editada por la Academia Argentina de Letras entre 2007 y 2013 con la aspiración de registrar el léxico de la oralidad en torno a diferentes actividades:

Ofrece al pueblo una colecta ordenada de las voces que toma de la boca viva de ese pueblo, de su cotidiana oralidad. Propone una serie de léxicos especiales, que recogen los vocablos y expresiones con los que el hombre

El ámbito aeronáutico admite también el uso genérico, pero es la posibilidad de la oposición la que otorga diferente valor a la expresión en el sistema léxico de especialidad. 
común se refiere a las realidades elementales de todos los días: el pan, el vino, el mate, la carne, el dinero, el fútbol, el colectivo; o con los que nombra los elementos de las artes y oficios en los que trabaja, como la carpintería, la telería, la tonelería, los dulces caseros.

Esta colección es un puente allegador entre la disciplina académica y la espontaneidad popular, entre la biblioteca erudita y la calle populosa, entre el saber libresco y la cultura oral. (Barcia, s/f, en línea).

Entre los volúmenes que integran esta colección se cuentan Léxico del automovilismo (2008) y Léxico del ciclismo (2008). Ambos resultan cercanos a la propuesta lexicográfica que presenta este artículo que se plantea como una sistematización del vocabulario cotidiano del transporte aéreo destinada a la consulta de personas que no requieren del mismo nivel de precisión que un especialista del campo.

Este trabajo sintetiza las decisiones lexicográficas adoptadas para su diseño, basadas en los aportes de Haensch y otros (1982), Porto Dapena (2002), Medina Guerra (2003). En términos específicos, describe la selección de los materiales empleados, la conformación de la base de datos léxica, las decisiones en torno a la macroestructura del texto y los criterios que operan en la selección y ordenamiento de los lemas, así como, por último, la microestructura de los artículos en preparación.

\subsection{El léxico del transporte aéreo}

Resulta casi un tópico hacer referencia al acelerado progreso del vuelo humano desde que la aerostación lo hiciera factible. En efecto, demostrados los principios fundamentales y desarrolladas las técnicas básicas que lo hicieron posible, las mejoras técnicas fueron incesantes y hoy contamos con la posibilidad de utilizar hasta aeronaves personales para pequeños usos cotidianos (drones) y se están proyectando vuelos rentados de "turismo espacial". La aspiración a volar explica las referencias plasmadas en diversas tradiciones literarias y en obras científicotécnicas previas al vuelo de los hermanos Montgolfier, en las que aparecen propuestas diversas e imaginativas de "máquinas voladoras". Sin embargo, recién con la experimentación sistemática se fue creando un léxico especializado y organizado también de manera sistemática. Este proceso es el mismo que se produce ante todo avance en el campo de la ciencia o de la técnica pero puede postularse que en su difusión social puede haber influido el marcado impacto social esta modalidad de transporte. La "locomoción aérea" se convirtió gradualmente en paradigma de la idea de progreso (Nisbet, 1981), en la que resultaban centrales los cambios en la relación del hombre con el mundo y sus distancias a través de otros medios de transporte como el tren y el transatlántico. 
En gran medida, las voces que hoy integran el léxico del transporte aéreo provienen de otras áreas del saber $y$, a través de procesos metafóricos o de especialización, vieron precisado su significado en el nuevo campo. Es el caso de las voces piloto, nave, timón o barquilla, todas ellas provenientes de la náutica. Además de abundantes neologismos, algunos abandonados (como muchadavi, propuesta para designar a las azafatas referida por Casares [1950]), también se adoptaron numerosas voces de otras lenguas, en especial del francés y del inglés (por caso, fuselaje, aterrizaje, flap, dron, entre otras), pero también del italiano y del alemán (por ejemplo, decolar y el epónimo zepelín).

Asociadas a significados precisos en los contextos situacionales profesionales, la importancia de su empleo terminológico es innegable para garantizar la seguridad lingüística de las operaciones aéreas (de- Matteis, 2010). Pero muchas de estas expresiones se constatan también en contextos de la vida cotidiana en los que se trata de aviación (en sentido literal o figurado) y en los que aparecen sin el valor preciso que tienen en la comunicación experta. Así, por ejemplo, en el discurso periodístico se registran imprecisiones al usar las expresiones alerón y flap. Mientras que, técnicamente, la primera designa una 'superficie de control de la aeronave que controla su movimiento sobre el eje longitudinal' y la segunda una 'superficie empleada para incrementar la sustentación que puede proporcionar un ala', su ubicación en el borde posterior de las alas de un avión explica que a veces se las use como equivalentes:

(01) La investigación judicial ha establecido que el accidente se produjo por una combinación de error humano y fallo técnico. Los pilotos olvidaron desplegar los alerones que facilitan que el avión se eleve en el despegue y ese olvido no fue advertido porque falló el mecanismo de alerta. (EP3 20120921, “Más seguridad aérea).

En otros casos, se registran diferencias entre las preferencias al interior o al exterior de las organizaciones aeronáuticas que no tienen que ver solo con la precisión referencial del léxico, sino con diferencias en su contenido axiológico. Como ejemplo puede considerarse la aceptabilidad de las voces avión y avioneta: mientras que cualquier 'avión de pequeño tamaño' es para los legos una avioneta, la misma aeronave es para la mayoría de los profesionales aeronáuticos un avión, y en las fuentes se constatan comentarios como el siguiente:

(02) P/D para los señores del Diario La Nacion [sic] que escriben sobre aviación NO llamen AVIONETA a un King Air 300 como el que lamentablemente tuvo el accidente en nordelta, es un avión hecho y derecho.

Los ejemplos de este trabajo se referencian mediante las siguientes claves: EP El país, España; LN: La Nación, Argentina. 
||En todo caso se lo podría denominar avión de pequeño porte, aeronave, aeroplano, etc, etc, etc, pero no avioneta. (LN 20140917: comentario de lector, "A pesar de los tres aviones caídos, este año habría menos accidentes aéreos que en $\left.2013^{\prime \prime}\right)$.

Para un profesional o un aficionado a la aviación, resulta despectiva la voz avioneta que, derivada del francés avionette, alude en su origen solo a una cuestión de tamaño (observable también en los pares léxicos ala-aleta, banca-banqueta, biciclobicicleta, camión-camioneta, moto-motoneta, patín-patineta). Estos hablantes perciben de manera negativa la superposición de sentidos que surge de los dos usos que tiene en español el sufijo de origen francés -ete, -eta (<-ette) pues también se emplea para derivar voces despectivas como los adjetivos regordete, calvete, etc. La asociación metafórica entre tamaño y complejidad de la operación de una aeronave explica la preferencia por avión, voz que jerarquiza al operador de una aeronave con independencia de su dimensión.

Avión y aeroplano, por otro lado, ilustran cambios diacrónicos en el léxico. Voces muy extendidas en los usos de un momento determinado pueden adquirir, en otro, un valor casi exclusivamente estilístico. Así, aeroplano presenta baja frecuencia en fuentes contemporáneas (el DEA considera su uso raro) y se emplea casi siempre para referirse a aeronaves propias de las primeras décadas del siglo $\mathrm{XX}$, evocando esta época:

(03) Escribo la palabra "aeroplano" y me quedo con la mirada perdida en la pantalla, donde una niebla blanca se disuelve lentamente arrastrada entre un rumor lejano de motores por la corriente de aire de las hélices. Aviones... (EP 20120805, Jacinto Antón, “La saga de los aviadores estrellados").

Por último, también pueden identificarse en la oralidad empleos figurados de voces aeronáuticas. Avión aparece en expresiones como "estar hecho un avión" o "ser un avión", con el sentido metafórico de 'ser rápido' o 'hacer una cosa velozmente' (deMatteis, 2005).

Las apropiaciones parciales de los significados técnicos que se traducen en imprecisiones referenciales, las diferencias en el sentido axiológico que poseen algunas voces según el contexto comunicativo, los cambios diacrónicos y los usos metafóricos (solo posibles por la integración de las voces en el léxico general) son fenómenos que interesan pues dan cuenta de procesos históricos y lingüísticos vinculados a esta modalidad de transporte y al sistema de la lengua. Un diccionario terminológico de la aviación oculta, evidentemente, estas dinámicas que, sin embargo, resultan reveladoras de cómo se enlazan los hechos lingüísticos con los factores histórico-sociales que los afectan. 


\subsection{Antecedentes}

\subsubsection{Estudios lingüísticos}

El léxico del transporte aéreo ha sido estudiado por especialistas en diversas lenguas, con variable grado de profundidad. Así, por ejemplo, el léxico francés de la aviación fue objeto de una exhaustiva tesis doctoral (Guilbert, 1965), mientras que las denominaciones para los vehículos aéreos en inglés también han sido consideradas con detalle por Stubelius (1958 y 1960) y, más recientemente, en un estudio que ha incorporado una perspectiva contrastiva entre francés, inglés y alemán (Viljanen, 2007).

Existen también estudios sobre el léxico español de varios tipos de locomoción. ${ }^{4}$ Para el caso del transporte aéreo, destacan los estudios de Vivanco Cervero (1999 y 2006) sobre los procedimientos de creación léxica que predominan en la terminología aeronáutica. También puede mencionarse el artículo de ÁlvarezAmandi y Uruburu (1988), muy anterior pero de menor alcance por centrarse en la formulación de una propuesta reactiva frente al empleo en español de préstamos de otras lenguas referidos al transporte aéreo. Sin embargo, ninguno de estos trabajos considera los usos léxicos en la comunidad no experta, como sí lo hacen algunos trabajos de de- Matteis (2005, 2009, 2011, 2012, 2014).

\subsubsection{Lexicografía}

Dejando de lado la lexicografía especializada, el espectro de materiales disponible justifica nuestra propuesta porque no hemos podido constatar la existencia de una obra que sistematice el léxico cotidiano de la aviación ni que dé cuenta de los hechos de variación registrados en las distintas comunidades hablantes de español. Aunque el fascículo 7 del primer volumen del Diccionario Histórico de la Lengua Española (DHLE) recoge muchas de las voces de uso frecuente para referirse a aspectos del transporte aéreo que comienzan con la letra $a$, hay que observar que algunas de ellas no se emplean más (es el caso, por ejemplo, de aerocarta). Otras muchas expresiones o acepciones propias de la aviación no aparecen recogidas en la lexicografía académica (por caso, decolar, que sí aparece en vox) o en otros diccionarios generales del español. Así, por ejemplo, la acepción aeronáutica de

Sobre el léxico ferroviario destacan los trabajos de Rodríguez Ortiz (por ejemplo, 2003), mientras que a los numerosos trabajos sobre el léxico de la navegación, que no mencionamos por falta de espacio, se suma el actual proyecto vinculado al desarrollo del NDHE dirigido por Congosto Martín (Proyecto "Nuevas Aportaciones al Léxico de la Navegación y la Gente de Mar (ss. XVI-XVIII)", Universidad de Sevilla). 
El léxico español del vuelo: un proyecto lexicográfico para el dominio del transporte aéreo

cesta, sinónimo de barquilla en el campo de la aerostación, y, por supuesto, de préstamos como low cost, que no pudimos constatar en ningún diccionariogeneral).

\section{El Léxico español del vuelo}

\subsection{Alcances y presupuestos}

El principal objetivo del proyecto del Léxico español del vuelo es dar cuenta del léxico aeronáutico registrado en el uso cotidiano de español en la actualidad, sin excluir voces desusadas o antiguas que dan cuenta de los cambios en este sector del vocabulario. El texto está destinado especialmente a un lector que, sin ser un profesional aeronáutico, puede requerir informaciones sobre el empleo cotidiano de voces aeronáuticas o que está interesado en su historia. Incluye, por tanto, voces empleadas para referirse a distintos aspectos del transporte aéreo: los vehículos, las personas, el vuelo, la infraestructura terrestre, el espacio aéreo, su comercialización, etc., para las que ofrece definiciones e informaciones sobre su historia y sus contextos de uso.

Desde un punto de vista teórico, como ya anticipamos, la propuesta se funda en los presupuestos de la teoría comunicativa de la terminología. Para esta perspectiva, una unidad léxica se convierte en palabra o término en condiciones pragmáticas que dependen de los usuarios que la emplean, las situaciones, los temas que vehiculiza y los tipos de discurso en los que aparece (Cabrè, 1999: 124- 125). Por lo tanto, los lemas del diccionario son unidades léxicas que, más allá de su potencial uso terminológico, se emplean o emplearon en forma oral o escrita y por fuera de los contextos profesionales para referirse al transporte aéreo.

Pese a la discusión teórica al respecto, El léxico español del vuelo asume como válida la posibilidad de la sinonimia, al menos en lo que hace al plano de la representación (Wolf, 1982: 349). En consecuencia, intenta dar cuenta de hechos de variación léxica registrados en este sector del léxico español en los ejes diatópico, diacrónico (desde el siglo XVIII) y diafásico. Para ello, más allá de contar con una estructura lexicográfica tradicional, la propuesta se vale de numerosos ejemplos cuya selección busca lograr que el lector sitúe las voces en sus contextos de uso habituales y que, al mismo tiempo y en la medida en que esto resulta posible, la historia de las palabras ilumine momentos y episodios representativos de la historia de la aviación.

\subsection{Alcance los materiales y criterios de selección}

Para relevar y seleccionar las voces se apeló a distintas fuentes primarias de amplia 
circulación social y representativas de distintas variedades del español, que se complementaron con diccionarios de carácter general como fuentes secundarias.

\subsubsection{Fuentes primarias}

Entre las fuentes primarias, se priorizaron los textos de la prensa escrita (semanarios, diarios y revistas), aunque se consideraron también algunos ejemplos del uso oral. Justifica esta prioridad la doble observación de que la prensa puede admitir muchos tipos de géneros textuales y de que, al mismo tiempo, todo ejemplar periodístico incluye las voces de distintos exponentes (profesionales o no) de la sociedad, (Bosque 2008: CLX-CLXII). Además, como señala Fritz (1988: 1623), los docentes y los medios "contribute to the popularization of scientific concepts and at the same time introduce semitechnical uses of the relevant vocabulary or trivializing translation equivalents". Por tanto, los datos léxicos obtenidos a partir de estas fuentes permiten estimar de manera acertada el grado de difusión e integración social de las voces en las comunidades bajo estudio. ${ }^{5}$ Los textos se obtuvieron tanto a partir de la consulta de archivos físicos ${ }^{6}$ como, sobre todo, a partir de los portales digitales de medios periodísticos de países de habla hispana, algunos con extensos archivos que cubren todo el siglo XX, y de diversas hemerotecas digitales. ${ }^{7}$

La tipología de los textos periodísticos analizados incluyó notas de interés general sobre aviación, crónicas de accidentes y seguimiento de las investigaciones subsiguientes, reportajes a personajes de interés, notas históricas y sobre acciones aéreas en conflictos bélicos, consejos para viajeros, humor gráfico, caricaturas e historietas de tema aeronáutico, etc. Algunas de las notas de interés general presentan descripciones técnicas más o menos detalladas y algunas pueden considerarse como textos de "divulgación técnico-científica". En estos casos, el uso de las comillas alerta sobre el carácter técnico o neológico de las voces pero elegimos no excluir las que, en otras oportunidades, aparecen sin explicaciones o definiciones.

5 Tomamos este concepto de Gimeno Menéndez (1990: 156), quien define la integración social de los préstamos como: "la frecuencia y difusión de un cierto elemento en el habla de la comunidad, y diacrónicamente como una fase del proceso del préstamo".

6 Deseamos reconocer la asistencia del personal de la Biblioteca Rivadavia (Bahía Blanca), Biblioteca Nacional (Buenos Aires), así como del Archivo del Museo Casa de los Tiros (Granada, España).

7 Algunas hemerotecas de prensa peninsular y la Hemeroteca Digital de la Biblioteca Nacional de España permiten acceder a ejemplares textuales de los siglos XIX y XX, incluyendo colecciones completas de medios americanos, por caso, del semanario argentino Caras y Caretas. En otros países, la digitalización tiene diversosalcances. 
Además de estos materiales, se han analizado textos de diversa naturaleza y procedencia. Estos incluyen algunas obras en prosa: relatos breves, cuentos, novelas y también biografías y autobiografías que reproducen o aluden a distintos aspectos de la actividad aeronáutica. A diferencia de Bosque, quien decide evitar la intencionalidad artística en la preparación de su diccionario, no descartamos los empleos lúdicos o creativos que se constatan en conversaciones de hablantes legos en aviación o en creaciones literarias pues consideramos que dan cuenta del grado de integración social de algunas voces.

También hemos atendido a otros textos orientados a un lector no especialista pero "cliente" del transporte aéreo, es decir textos producidos desde las organizaciones aeronáuticas y destinados a los usuarios de sus servicios. Así, se han considerado publicidades gráficas de compañías aéreas, publicaciones del tipo organ-house (deMatteis, 2011) y folletos de información aeroportuaria. En este mismo sentido y dando lugar también a manifestaciones textuales que caracterizan la sincronía del siglo XXI, se incluyen voces registradas en entradas publicadas dentro de espacios interactivos (blogs y foros de Internet abiertos a las personas interesadas 0 aficionadas a la aeronáutica). En estos casos se ficharon unidades léxicas que, si bien pueden ser de uso terminológico o jergal en el ámbito endo-institucional (deMatteis, 2009), interesan y son definidas para beneficio de lectores ajenos a estos ámbitos. En estos espacios de interacción, los comentarios de hablantes sin formación aeronáutica permiten identificar los significados cotidianos que se atribuyen a las distintas unidades léxicas. Contribuciones como la siguiente presentan incluso afirmaciones sobre el uso "correcto" de las palabras que señalan la distancia entre los usos cotidianos y los terminológicos de las unidades léxicas:

(04) Se dice "abortar" el despegue y hacer "escape" al aterrizar. (LN 20120213: comentario de lector, “Dos aviones se rozaron en Aeroparque, pero no llegaron a chocar").

Asimismo, se han consultado y fichado algunas obras de carácter divulgativo de distintas etapas históricas, que operan como fuentes primarias complementarias por incluir entre sus destinatarios a lectores no especializados. En el interés de ampliar el corpus correspondiente a los siglos XVIII y XIX y, aunque no reflejan precisamente usos cotidianos de la lengua, también hemos considerado excepcionalmente algunas propuestas de patente de etapas muy iniciales del transporte aéreo relevantes para indicar etapas en la historia de este vocabulario.

Entre las fuentes primarias, apelamos en forma complementaria a datos del Corpus Diacrónico del Español (CORDE), del Corpus de Referencia del Español Actual (CREA), del Corpus del Español del Siglo XXI (CORPES XXI) y del Corpus de Español de Davies. 
Por último, en lo que hace al registro de usos oral, se asentaron los datos contextuales, la variedad lingüística y se estimaron los perfiles sociolingüísticos de los hablantes.

\subsubsection{Fuentes secundarias}

Como fuentes secundarias recurrimos a un amplio conjunto de obras lexicográficas sobre la lengua española que incluye, entre otros, el DRAE -ahora DEL-), a otros diccionarios generales como los de Seco (1999), Cayuela (2002), Moliner (2007), a diccionarios de americanismos y argentinismos, como los de Haensch y Werner (2000) y Academia Argentina de Letras (2008).

\subsection{Base de datos}

El procesamiento de los textos periodísticos que constituyen la mayor parte de nuestras fuentes primarias, ha sido diferente en función del formato digital del documento consultado, esto es textos digitales en portales de consulta vigente (formato .htm, por ejemplo) o textos digitalizados en hemerotecas o archivos en línea (formato .pdf o imagen, con diverso grado de reconocimiento de caracteres) o, por último, textos en papel, consultados en bibliotecas y archivos físicos, que fueron fotografiados y archivados como .jpg.

El primer formato corresponde, de manera general, a todos los textos periodísticos posteriores al año 2000, fecha que tomamos como punto de partida de la mayoría de los diarios digitales de acuerdo con las posibilidades que ofrece la consulta histórica de estos portales. En este caso, se los descargó y convirtió en todos los casos a formato .txt. Estos archivos fueron luego procesados con las herramientas de WordSmith Tools, para generar una lista de palabras que orientó la primera selección de lemas. Los textos correspondientes a los siglos XVIII, XIX y XX en formato .pdf o imagen fueron fichados de manera manual y se agregaron las voces faltantes de acuerdo también con el criterio de frecuencia de uso.

En todos los casos, las voces fueron sistematizadas en una base de datos léxica diseñada con la herramienta Toolbox, en la que se consignaron las diferentes informaciones necesarias para elaborar los artículos lexicográficos. 


\section{Macroestructura}

\subsection{Selección de lemas}

El diccionario intenta incorporar el máximo número de expresiones vinculadas al transporte aéreo que aparecen en nuestras fuentes primarias. Solo se excluyen aquellas voces para las que simultáneamente se dan estas tres condiciones: muy baja frecuencia de uso actual ( $\leq 5$ ocurrencias en el corpus de prensa escrita digitalizado), presencia constante de indicadores de su carácter terminológico en todas las fuentes (destacado en itálicas, negritas o comillas, presencia de definiciones o explicaciones) y alto nivel de especificidad. A sí, por ejemplo, si consideramos las denominaciones para instrumentos usados en vuelo, se incluyen la voz altímetro y la sigla ACARS (v. infra). Ambas presentan baja frecuencia y pueden aparecer, en algunas ocasiones, definidas, por lo que se las incluye con la marca de tecnicismos para indicar que su uso es más frecuente entre los profesionales. En cambio, no se incluyen los sintagmas escala barométrica ('manera en la que se mide y configura la información de un altímetro') ni unidad de gestión (uno de los componentes del sistema ACARS) por presentar un elevado nivel de especificidad.

Se admiten también los neologismos y algunos elementos jergales registrados en comunidades de aficionados como, por ejemplo, aerotrastornado ('persona muy aficionada a la aviación'). De acuerdo con el carácter globalizante e internacional del transporte aéreo, se asume una perspectiva abierta e inclusiva frente a los préstamos frecuentes, estén o no adaptados a las pautas morfológicas y fonológicas del español, como boarding pass ('pase de abordaje'), low cost ('de bajo costo') u overbooking ('sobreventa'), para solo mencionar algunos ejemplos.

Asimismo, se incluyen siglas habituales para denominar organismos, equipos e, incluso, condiciones de las operaciones aéreas. En este caso, se incorporan tanto las de origen español (OACl, 'Organización de la Aviación Civil Internacional') como de préstamo (ILS, del inglés 'Instrument Landing System', ACARS 'Aircraft Communications, Addressing and Reporting System' o CAVOK 'Ceiling and Visibility OK').

Algunos afijos muy productivos en este vocabulario (prefijos como aero- o astro- $y$ sufijos como - nauta o -aje) se registran en entradas independientes. Por último, a diferencia de otros diccionarios, las letras tienen entrada solo a través de las unidades léxicas empleadas en la radiotelefonía aeronáutica como representación desambiguadora de las letras del alfabeto (alfa, bravo, charli, etc.).

Como es habitual en la tradición lexicográfica, los lemas se presentan en una 
posición destacada en negritas y con una fuente de tamaño superior. ${ }^{8}$ Su ordenamiento alfabético es directo. Se siguen las prácticas normales para la presentación de adjetivos y verbos, mientras que, en lo que hace a los sustantivos que presentan variabilidad genérica, de acuerdo con las convenciones habituales de la lexicografía española se los presenta por la forma del masculino y femenino singular. Esta aproximación se mantiene, aun si la forma más frecuente en el uso es la femenina, como ocurre en el caso de las designaciones para profesiones con prevalencia del personal femenino (v. infra).

aeromóvil. I (De aero- y móvil). adj. Mil. Dicho de una fuerza militar que se transporta por vía aérea. Fin 20100826: El acto fue realizado por integrantes de la Fuerza Aérea Argentina pertenecientes al I Escuadrón Aeromóvil autodenominado Avutardas Salvajes, uno de los dos escuadrones que tuvo su base de operaciones en Río Grande para la defensa de la ciudad en el conflicto bélico de 1982 por las Islas Malvinas. ("Homenaje a caídos mártires de la Fuerza Aérea Argentina en la guerra de Malvinas").

II m. p. us. aeronave.

ABC Co 20001006 [CREA]: El piloto luqueño Marcos Maldonado tendrá para esta edición del Transchaco Rally 2000 a un copiloto que conoce bastante del territorio y la competencia chaqueña, pero desde el aire. Johnny Zeballos, quien desde hace ya varios años

\begin{abstract}
viene realizando la misma tarea, informar desde el aeromóvil para una de las emisoras que trasmiten la competencia, estará sentado en la butaca derecha del VW Gol GL 1600 de la clase A 6. ("Restan pocas horas para un nuevo rally del Chaco"). CYC, 1911, ํ691: El aeromóvil "Gaviota" (título de nota).

OBS.: Aunque el sustantivo fue recogido por el RAE U 1936 como "Aeronave o avión", que es la misma definición que proporciona el DLE, y aunque también es registrado por el VOX y por el DEA - con la marca de raro en este caso-, el sustantivo se utilizaba con mayor frecuencia en las primeras décadas del siglo XX para dar cuenta de distintos vehículos que eran diseñados por los inventores y constructores del momento. En la actualidad se registra sobre todo el adjetivo.
\end{abstract}

Figura 1. Artículo lexicográfico para aeromóvil.

Por último, la homonimia se resuelve en la misma entrada, como en el caso de aeromóvil (figura 1), presentando primero las formas adjetivas y luego las sustantivas de acuerdo con el modelo del $D E A$, que las separa con números romanos ennegrita.

\subsection{Ordenamiento de las subentradas}

A la fecha, las entradas del léxico incluyen unos 1400 lemas, algunos de ellos con diversas subentradas. Así, por ejemplo, para el lema aeropuerto algunas colocaciones nominales de forma SUST + ADJ son alternativo, civil, internacional, militar, o privado, incluidas por su frecuencia de uso. Otras colocaciones nominales de forma SUSTANTIVO + COMPLEMENTO son $\sim$ de cabotaje, $\sim$ de carga, $\sim$ de destino, $\sim$ de embarque, $\sim$ de llegada, $\sim$ de origen, $\sim$ de partida y $\sim$ de socorro. Entre las subentradas de otras voces se incluyen también usos figurados como bajar los flaps, pedir pista, volar alto.

Por limitaciones de espacio, los artículos seleccionados como ilustración omiten o reducen el número de ejemplos y observaciones que constan en el diccionario. 


\section{Microestructura}

La estructura del artículo lexicográfico se organiza de acuerdo con el siguiente modelo: lema, etimología, información gramatical, definición, remisiones internas. A cada expresión la acompaña también un conjunto ilustrativo de ejemplos de uso y, si resulta pertinente, observaciones sobre distintos aspectos que se consideran significativos, como mostramos en el caso de aeromóvil. Otras informaciones que se consignan cuando resultan necesarias son las fuentes de la información etimológica y de la definición, los alternantes gráficos desusados o vigentes, informaciones sobre la realización oral más corriente de préstamos y siglas.

\subsection{La información etimológica}

Siempre que es posible, la presentación de la información etimológica recupera las informaciones de obras lexicográficas previas y solo en escasas ocasiones se postulan etimologías elaboradas sobre la base de estudios léxicos propios. Cuando la etimología es textual de una única fuente, esta se indica entre corchetes y de acuerdo a las claves convencionales para referir a las obras lexicográficas. Caso contrario, se trata de una elaboración propia sobre la base de distintas entradas en el mismo diccionario, de distintas obras o lexicográficas o de la elaboración de los datos. Como se reconoce en el texto, la fuente más utilizada para las etimologías está dada por las distintas ediciones del DRAE-ahora el DLE-, pero como en estos diccionarios la etimología de la voz puede encontrarse separada en distintas entradas, la información se unifica sin dejar de reconocer la fuente, como en el ejemplo de aeromozo, a (figura 2).

aeromozo, -a. (De aero- y moza, y esta de or. inc.[DLE]) aero-mozo. m. y f. Am. Exo. Azafato o azafata de aviación. $\rightarrow$ azafato, a; aeromozo, a; auxiliar. $\sim$ de vuelo; cabinera; camarero, a; comisario, a. $\sim$ de vuelo; hostess; jefe, a. de cabina; sobrecargo; steward, -ess; TCP; tripulante. $\sim$ de cabina de pasajeros.

TCP. (Acrón., tripulante de cabina de pasajeros, pronunc. corriente /te-se-pé/). com. Endo. Miembro de una tripulación que vela por la seguridad de sus pasajeros al tiempo que se ocupa de su comodidad y atención, representando a la compañía a la que pertenece. $\rightarrow$ azafato, a; aeromozo, a; auxiliar. $\sim$ de vuelo; cabinera; camarero, a; comisario, a. de vuelo; hostess; jefe, a. de cabina; sobrecargo; steward, -ess; tripulante. $\sim$ de cabina de pasajeros.

hostess. (Angl., pronunc. corriente /xóstes/). f. Desus. Azafata. [DEA]. Frec. preced. por air o flight $\quad \rightarrow$ azafato, a; aeromozo, a; auxiliar. $\sim$ de vuelo; cabinera; camarero, a; comisario, a. $\sim$ de vuelo; jefe, a. de cabina; sobrecargo; steward, -ess; TCP; tripulante. $\sim$ de cabina de pasajeros.

Figura 2. Artículo lexicográfico para aeromozo, a, TCP y hostess.

En el caso de las siglas, como $T C P$, en lugar de la etimología se presenta el despliegue de la expresión (figura 2). Tras su definición, además, se indica la realización oral más 
habitual, siguiendo para ello el modelo del $D E A$, práctica que también se sigue en el caso de las voces de préstamo, como hostess. Como puede verse en estos casos, las remisiones contribuyen a apreciar el número de expresiones alternativas y semánticamente relacionadas.

\subsection{La definición}

Cuando resulta factible, se apela a las definiciones de diccionarios que hayan registrado la voz. En ocasiones, sin embargo, estas definiciones se consideraron insuficientes para un diccionario centrado en el léxico del vuelo y se las modificó incorporando aspectos semánticos que precisan los valores detectados en el uso cotidiano y, si corresponde, profesional de las expresiones.

Cuando la definición se toma de forma textual de algún diccionario, se indica la fuente según claves convencionales. Si consideramos el caso de aeromozo, $a$, el DLE la define como 'Azafato de aviación', utilizando solo la forma masculina. Así, si bien seguimos la norma de uniformar la presentación de los sustantivos por la forma masculina, en la definición optamos por dar cuenta de ambos géneros, sobre todo en la medida que la demografía de este colectivo demuestra que la mayoría de las personas que desempeñan esta profesión son de género femenino. Por este motivo, no aparece referenciada la fuente en la definición. En cambio, en el caso de hostess, la encontramos definida en el DEA y se da cuenta de esto entre corchetes. Cuando esta referencia no consta se trata de una definición propia o que adapta las de un conjunto diverso de obras lexicográficas (figura 2).

\subsection{Marcas}

La marcación de las voces se rige por las pautas habituales en la lexicografía del español, introduciendo mínimas modificaciones vinculadas con el registro de la variación diafásica. En las siguientes secciones se sintetizan los principales criterios utilizados.

\subsubsection{Variación diafásica}

En el artículo de la voz aeromozo, a y de la sigla TCP en la sección precedente, puede observarse una marcación particular, que alude a los contextos de uso en el que cada expresión es preferida. Las voces marcadas como Exo. (exoinstitucionales) son las que operan en el habla cotidiana, mientras que las expresiones indicadas como Endo. (endoinstitucionales) son las preferidas al seno de las organizaciones aéreas. Como se señala en de- Matteis (2014), la expresión TCP busca jerarquizar la 
profesión, reconociendo todas las tareas que cumple un tripulante de cabina: las de seguridad, las de atención y las de representación. En este sentido, tiene un significado mucho más preciso que justifica su preferencia dentro de las organizaciones aéreas. Pero son, sobre todo, las diferentes connotaciones axiológicas percibidas por los hablantes las que justifican su marcación como endo o exo-institucionales. Frente a TCP se ubican, por ejemplo, aeromozo, a pero también azafato, a - la que presenta mayor frecuencia de uso-, o cabinera (figura 3), que se marcan como Exo, en la medida en que son preferidas por los hablantes ajenos al mundo aeronáutico y explícitamente rechazadas por los profesionales. La ausencia de esta marca señala que la expresión resulta admisible en ambos contextos sociales. También dentro de la variación diafásica, se marcan elementos jergales (Ver.), es decir, voces que no necesariamente se usan en la comunicación cotidiana pero que aparecen en los comentarios a notas periodísticas de personas aficionadas a la aviación o en cuentas de redes sociales dedicadas a actividades recreativas vinculadas a la aviación, como por ejemplo (sublo, 'tipo de pasaje liberado, sujeto a carga -del inglés, 'subject to load'- y, por extensión, la persona que viaja con este pasaje'; spotter, 'persona aficionada a tomar fotografías a aviones' o livery, 'esquema de pintura de una aeronave').

Por último, se emplea también la marca de tecnicismo (Tec.), para aquellas voces que cumplen los criterios señalados en la sección 3.1. Además, se precisan marcas para voces de otras disciplinas, como cirro, de la Meteorología (Met.), o abordaje, que corresponde a la esfera del Derecho Aeronáutico (Der.).

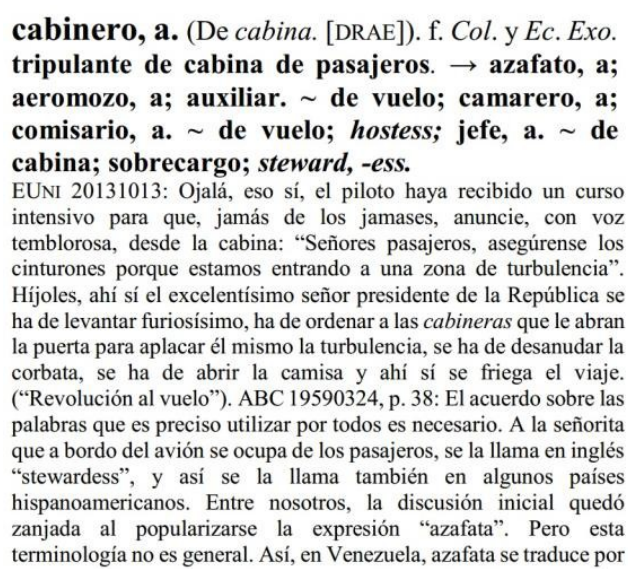

"aeromoza", y en Colombia, por "cabinera". ("Defensa del español").

decolaje. (Del fr. décollage, y esta de décoller 'despegar'). decolage. m. Am. despegue.

ELME 20130209: Estos son los minutos más pavorosos que se haya filmado en mucho tiempo acerca de un decolaje en malas condiciones (...) ("El vuelo). LRA, 19581110, p.6: El pesado avión comienza a moverse. Las luces azules demarcatorias de la pista pasan cada vez con mayor rapidez. Recorre unos 1.500 metros y se detiene. Empiezan a calentarse los motores hasta alcanzar el número de revoluciones necesarias para efectuar el decolaje.

rodadura. (Del fr. décollage, y esta de décoller 'despegar'). m. Esp. carreteo. $\rightarrow$ rodaje. LV 20140929: Uno de sus miembros captó con su objetivo el pasado verano cómo el piloto de un avión abortaba un aterrizaje al detectar otra aeronave haciendo maniobras de rodadura en la zona para encaminarse hacia la pista de despegue.("El Prat se pone de moda entre los 'spotters' europeos").

Figura 3. Artículos lexicográficos para cabinero, a, decolaje y rodadura. 


\subsubsection{Variación diatópica}

Como es habitual en los diccionarios generales, se emplean marcas de variación diatópica que aluden a regiones de habla hispana (por ejemplo, Am. América, Centroam. Centroamérica) o a países específicos: Arg. (Argentina); Bol. (Bolivia); Chi. (Chile); Col. (Colombia); Ec. (Ecuador); Esp. (España); Méx. (México) o Per. (Perú); etc. Algunos ejemplos de su empleo son los que afectan a las voces cabinero, $a$, decolaje y rodadura (figura 3 ).

\subsubsection{Variación diacrónica y frecuencia de uso}

Aunque la frecuencia de uso en nuestro corpus no puede considerarse un indicador absoluto con respecto a la presencia de estas voces en la comunicación cotidiana, puesto que trabajamos con fuentes que se ocupan de temas aeronáuticos y esto introduce un evidente sesgo, constituye un elemento de juicio imprescindible para la selección de los lemas a incluir y para el análisis de la variación diacrónica. Consideramos que combinando la frecuencia de las voces en nuestro corpus digital con la datación de los ejemplos, puede contarse con una primera pauta orientadora para definir la pertinencia de marcas diacrónicas (tabla 1).

\begin{tabular}{llll}
\hline Ocurrencias & $\begin{array}{l}\text { Frecuencia relativa } \\
(\%)^{*}\end{array}$ & $\begin{array}{l}\text { Datación de } \\
\text { ejemplos }\end{array}$ & $\begin{array}{l}\text { Marca dia- } \\
\text { crónica }\end{array}$ \\
\hline 0 & - & s. XVIII & Ant. \\
\hline 0 & - & s. XIX & Ant. \\
\hline 0 & - & s. XX (h. 1975) & Desus. \\
\hline$\leq 10$ & - & s. XXI & Desus./P. us. \\
\hline$\leq 250$ & $\leq 0.02$ & s. XXI & ¿P. us.? \\
\hline$\leq 500$ & $\leq 0.04$ & s. XXI & Sin marca \\
\hline$\leq 750$ & $\leq 0.06$ & s. XXI & Sin marca \\
\hline$\leq 1000$ & $\leq 0.08$ & s. XXI & Sin marca \\
\hline$>1000^{9}$ & $\geq 0.08$ & s. XXI & Sin marca \\
\hline
\end{tabular}

Tabla 1. Escala de frecuencias de uso y datación de registros.

*Valores de referencia: número de textos de prensa escrita posteriores al año 2000: 1520; tokens: 1185668, types: 55884, StTTR: 43,08. Última actualización: 15/03/2016.

Según la combinación de criterios frecuencia y datación, se consideran antiguas las voces que solo se registran en los siglos XVIII o XIX (airestación, airestacionar 'mantenerse fijo en el aire', aeronautación 'aeronavegación', aereodinámico, a). Si, en

Muy pocos lemas (avión, vuelo, piloto, aeropuerto, aerolínea, compañía, pasajero/a, accidente, seguridad, aviación, pista, además del adjetivo aéreo/) superan las 1000 ocurrencias. 
cambio, las unidades fueron detectadas en el fichaje de textos correspondientes al siglo XX, hasta aproximadamente el año 1975, y no aparecen en fuentes posteriores al año 2000 se las considera como desusadas. Algunos ejemplos que pueden mencionarse en este grupo son purser o hostess, como alternativas para azafata.

Ahora bien, es necesario reconocer que los criterios adoptados son discutibles y, en ocasiones, se ha debido recurrir a una apreciación menos rigurosa de los datos cuantitativos y a una evaluación cualitativa de los ejemplos de uso, intentando también relevar, en lo posible, el juicio de los hablantes. Así, la marca de desusada también se aplica a algunas voces que pueden aparecer en la prensa escrita sincrónica con una frecuencia despreciable (por ejemplo, inferior a las 10 veces) y en textos que intentan recrear una época específica de la aviación. Además de aeroplano, otros ejemplos son volación ('aviación') o volador ('piloto de aeroplano'). Para expresiones que presentan frecuencias superiores en la sincronía, pero que, sin embargo, se mantienen por debajo de las 250 ocurrencias el corpus de textos digitales, se analiza con detalle cada caso particular y, en función de su aparición o no en otros textos anteriores al año 2000, se define si se trata de un fenómeno asociado a la vigencia diacrónica de la expresión -esto es, de una expresión poco usada como las mencionadas- o, por el contrario, de un hecho de variación diafásica. Ejemplos de este último tipo serían los tecnicismos como altímetro o los elementos jergales (v. infra).

\subsection{Formas gráficas alternativas}

Las formas gráficas desusadas se presentan con una fuente un tamaño menor en negrita, siguiendo el modelo del DHLE. Es el caso, por ejemplo, de las grafías decolage y aereopuerto o aéreopuerto, estos últimos alternantes (figura 4).

$$
\begin{aligned}
& \text { aeropuerto. (De aero- y puerto). aereopuerto; } \\
& \text { aéreopuerto. m. Superficie extensa de terreno con } \\
& \text { pistas y calles adecuadas para la operación comercial }
\end{aligned}
$$

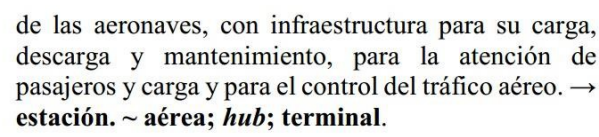
pasajeros y carga y para el control del tráfico aéreo. $\rightarrow$ estación. aérea; hub; terminal.

Figura 4. Artículo lexicográfico para aeropuerto.

Las voces que en su empleo actual poseen diferente representación gráfica se ordenan de acuerdo con el lugar alfabético que les corresponde: así, por ejemplo, si bien abrevalijas es más frecuente en nuestro corpus, la precede en el diccionario la entrada abre valijas. Por otra parte, el artículo dedicado a la forma más frecuente hace referencia a la alternativa ortográfica, precediéndola con el signo $\diamond$ (figura 5). 
abre valijas. (De abrir y valijas). com. Arg. abrevalijas. $U$. en $\mathrm{pl}$.

LN 20140112: Según cuestionó, "estos reclamos de unos pocos responden a intereses particulares de un grupo de delegados que pretende mantener un esquema que hoy es ineficiente" y apuntó contra "los grupos mafiosos de los 'abre valijas', personal de rampa que roba el equipaje de los pasajeros". ("Por un conflicto gremial con los maleteros, hubo cancelaciones y demoras de vuelos de Aerolineas Argentinas"). abrevalijas. (De abrir y valijas). com. Arg. Personal de un aeropuerto que aprovecha su acceso al equipaje para realizar hurtos. U. en $\mathrm{pl}$. $\diamond$ abre valijas. LN 20140508: La permanente reaparición de las bandas de los denominados "abrevalijas" y la impunidad con la que se desempeñan en las dos terminales permiten sospechar tanto la connivencia de cierto nivel jerárquico como graves fallas de seguridad en sectores sensibles que deberían estar sometidos a la vigilancia de las cámaras las 24 horas. ("Abrevalijas: un delito recurrente").

Figura 5. Artículo lexicográfico para abre valijas.

\subsection{Los ejemplos de uso}

La selección de los ejemplos busca ofrecer al lector modelos de uso de las diferentes acepciones de una voz, pero también ilustrar algunos de los cambios semánticos y pragmáticos constatados. Casi siempre se ofrecen de al menos dos variedades lingüísticas del español, aunque las variedades más representadas, sin embargo, son las del español de Argentina y de España.

Como se muestra en el artículo para acuatizaje (figura 6), se priorizan ejemplos que: a) sugieren definiciones parciales o completas o que presentan analogías, traducciones (si se trata de voces de préstamo) o explicaciones que, junto a precisar el significado de las voces, ponen en evidencia el rol de difusión social de términos aeronáuticos por parte de la prensa y b) mostrar los avances aeronáuticos y la consiguiente incorporación de voces nuevas en el léxico español. Se busca, de esta manera, que el lector acceda, simultáneamente, a la integración de este léxico en el sistema de la lengua y a los momentos, hechos y personajes de la historia aeronáutica que marcaron su contexto de formación. Con este fin, los fragmentos seleccionados se ordenan dentro del artículo en orden cronológico inverso: el más actual para acuatizaje corresponde al español peninsular ${ }^{10}$-diario $A B C$, edición de Sevilla (ABC-S)-, el segundo al español de Argentina -diario Clarín (CL). El texto peninsular de La Libertad (LL) se remonta al año 1926 e ilustra las discusiones peninsulares en torno a la selección de la forma léxica más apropiada para denotar la 'acción y efecto de posarse en el agua que realiza una aeronave'. La observación que sigue a los ejemplos, los interpreta y explica en el contexto histórico de la introducción de la voz, en este caso, como neologismo que permite evitar la influencia del francés sobre el español. Esta observación, al mismo tiempo, ilumina las relaciones entre lenguas y tecnologías, en una etapa -la de la primera posguerrade gran difusión del transporte aéreo.

10 En la presentación de la fuente de los ejemplos se emplea el código asignado más la fecha en formato año-mes- día (por ej., LN 19910205: [...]), con variaciones si el texto proviene de corpus digitales o de otro diccionario. Para la publicación se gestionarán los permisos correspondientes con los distintos medios periodísticos. 


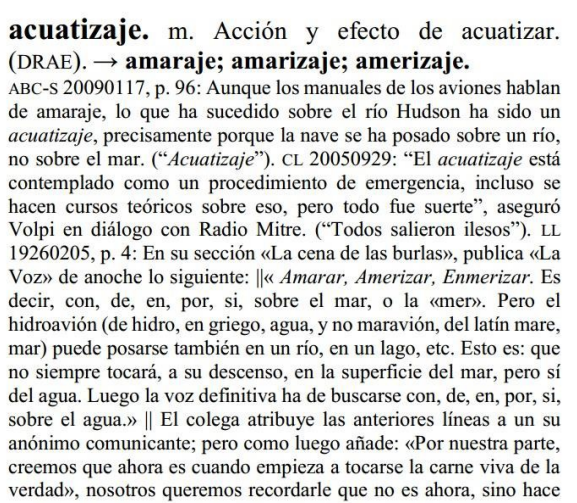

acuatizaje. m. Acción y efecto de acuatizar. ABC-S 20090117, p. 96: Aunque los manuales de los aviones habla no sobre el ma " "A poie") contemplo ( Acualzaje"). CL 2005029. "El acuanizaje es. ( nerizar. hidroavión (de hidro, en griego, agua, y no maravion, del latin mare, mar) puede posarse también en un rio, en un lago, etc. Esto es: que tocará, a su descenso, en la superficie del mar, pero sobre el agua.» El colega atribuye las anteriores lineas a un verdad», nosotros queremos recordarle que no es ahora, sino hace de amaraje, lo que ha sucedido sobre el río Hudson ha sido un

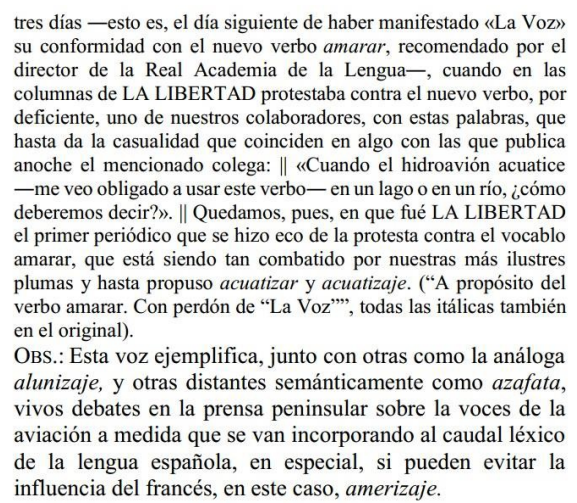

Figura 6. Artículo lexicográfico para acuatizaje.

\section{Comentario final}

Si, retomando los conceptos de Gómez de Enterría Sánchez, consideramos nuevamente el doble camino que recorren algunas unidades léxicas, entre el léxico general y el especializado, nos encontramos con que esta situación no siempre es reflejada en la lexicografía de una lengua: mientras que la especialización del léxico general puede consultarse en los diccionarios terminológicos, no hay, en cambio, muchos materiales -fuera de estudios lingüísticos específicos- que recorran el camino inverso y den cuenta de la penetración de las unidades especializadas de un ámbito determinado en la lengua general. En consecuencia, el hablante ajeno al ámbito de especialidad solo puede informarse recurriendo a una obra que no lo tiene como destinatario prioritario, con las dificultades que esto puede entrañar. En este contexto, El léxico español del vuelo atiende a salvar esta carencia en el caso del transporte aéreo, intentando registrar y definir de manera clara y sin caer en un excesivo nivel de detalle técnico aquellas voces más frecuentes en los usos cotidianos de distintas comunidades de habla española. Consideramos que, cuando tenga su forma definitiva, el material resultará de interés para el no especialista, pero también para usuarios como los docentes de español como lengua extranjera o los periodistas que requieran acercarse a este vocabulario por un interés profesional no técnico, pero también, en una perspectiva especializada centrada en la diacronía, para los historiadores de la aviación y del transporte.

\section{Acerca de la autora}

Lorena M. A. De- Matteis es Licenciada y Doctora en Letras, ambas titulaciones con orientación en Lingüística, por la Universidad Nacional del Sur (UNS, 2001 y 
2006). Ganadora del Primer Premio a Tesis de Doctorado otorgado por la Asociación Latinoamericana de Estudios del Discurso (2007). Es Investigadora Adjunta del Consejo Nacional de Investigaciones Científicas y Técnicas (CONICET). Se ha especializado en el análisis del discurso institucional de entornos sociotécnicos de alto riesgo. Ha participado y dirigido proyectos de investigación lingüística financiados por la Secretaría General de Ciencia y Tecnología de la UNS y actualmente integra, asimismo, un PIO financiado por CONICET. Ha desarrollado pasantías de investigación en el Instituto de Lingüística de la Universidad Nacional de Buenos Aires (2005), el Instituto Nacional de Medicina Aeronáutica y Espacial de Argentina (INMAE, 2005) y la Facultad de Filosofía y Letras de la Universidad de Granada (2008 y 2013). Ha publicado artículos en revistas nacionales e internacionales, así como un libro sobre la comunicación institucional en ámbito aeronáutico.

\section{Agradecimientos}

Hasta la fecha, este proyecto se ha desarrollado en el marco de sucesivos Proyectos de Grupos de Investigación financiados por la Secretaría General de Ciencia y Tecnología de la Universidad Nacional del Sur, como así también por los programas de becas para jóvenes docentes investigadores COIMBRA (2008) y las becas externas para investigadores asistentes de CONICET (2013).

\section{Recepción del artículo}

Fecha de recepción del artículo: 26 de marzo de 2017

Fecha de recepción de la versión modificada y aceptación del artículo: 11 de noviembre de 2017

\section{References}

Academia Argentina de Letras (2008). Diccionario del Habla de los Argentinos. Buenos Aires: Emecé Editores.

Álvarez-Amandi, C. \& Uruburu, A. (1988). Análisis semántico del lenguaje de la aviación. En M. V. Romero, F. Lautre \& C. Saralegui (Eds.), Actas del $5^{\circ}$ Congreso Nacional de Lingüística Aplicada. La enseñanza de la lengua con fines específicos (pp. 101-09). Pamplona: Universidad de Navarra y Asociación Española de Lingüística Aplicada (AESLA). 
Barcia, P. L. (s/f) Prólogo de la Colección «La Academia y la lengua del pueblo». < $\underline{\text { http://www. }}$ aal.edu.ar/?q=node/192>. [5/4/2017].

Bosque, I. (Dir.) (2008). REDES. Diccionario combinatorio del español contemporáneo. Madrid: Ediciones SM.

Cabrè, Ma. T. (1999). La terminología: representación y comunicación. Elementos para una teoría de base comunicativa y otros artículos. Barcelona: Institut Universitari de Lingüística Aplicada.

Cabrè, Ma. T. \& J. Gómez de Enterría (2006). La enseñanza de los lenguajes de especialidad. La simulación global. Madrid: Gredos.

Casares, J. (1950). Punto redondo. Diario ABC, 24/6/1950, p. 3.

Cayuela, N. (2002). Diccionario de uso del español de América y España. Barcelona: VOX.

de- Matteis, L. M. A. (2005) Entre "Zeppelines" y "Boeings 747": metáforas del vuelo y la aviación en el habla cotidiana en español bonaerense. Literatura y Lingüística, 16, 221245. <http://www.scielo.cl/pdf/lyl/n25/art13.pdf> [5/4/2017].

(2009). Mito y ciencia en la representación discursiva de los inventores de globos aerostáticos y de los primeros aeronautas en textos de hablantes de español peninsular. Lingüística y Literatura, 56, 126-154. <http://aprendeenlinea.udea.edu.co/revistas/index. php/lyl/article/view/5174> [5/4/2017].

(2010). Sobre el concepto de seguridad lingüística: propuesta de formulación para contextos institucionales específicos. Tonos Digital, 19. <http://www.um.es/tonosdigital> [5/4/2017].

(2011). Aviación y volación: un caso de variación léxica entre el español peninsular y el español bonaerense a principios del siglo XX. En Rojas Mayer, E. M. (Coord.), Léxico e Interculturalidad: nuevas perspectivas (pp. 403-19). Tucumán: Instituto de Investigaciones Lingüísticas y Literarias Hispanoamericanas, Universidad Nacional de Tucumán.

(2012). Expresiones de origen náutico en el nacimiento de las distintas formas de volar: aerostación, aviación y astronáutica. Literatura y Lingüística, 25, 275-298. <http://www. scielo.cl/scielo.php?script=sci arttext\&pid=S0716-58112012000100013> [5/4/2017].

(2014). «Señoritas en busca de nombre»: jerarquización de una profesión a través del léxico. Revista de Lexicografía XX, 77-106.

Departamento de Investigaciones Lingüísticas y Filológicas (2008). Léxico del automóvil. Buenos Aires: Academia Argentina de Letras.

Estopá, R. (1998). El léxico especializado en los diccionarios de la lengua general: las marcas temáticas. Revista Española de Lingüística, 28(2), 359-387.

Fontanella de Weinberg, Ma. B. (1995). El español de América. Madrid: Mapfre.

Fritz, G. (1988). Cambio de significado y cambio de vocabulario. En Ammon, U, Dittmar, N. \& K. Mattheier (Eds.), Sociolinguistics (II, pp. 1614-31). Berlín: Walter de Gruyter.

Gómez de Enterría Sánchez, J. (2009). El español lengua de especialidad: enseñanza y 
aprendizaje. Madrid: Arco/Libros.

Guilbert, L. (1965). La formation du vocabulaire de l'aviation. Paris: Larousse.

Haensch, G. \& R. Werner (2000). Diccionario del español de Argentina. Madrid: Gredos.

Haensch, G., Wolf, L., Ettinger, S. \& Werner, R. (1982) La lexicografía: de la lingüística teórica a la lexicografía práctica. Madrid: Gredos.

Medina Guerra, A. (Coord.) (2003). Lexicografia española. Barcelona: Ariel. Moliner, M. (2007). Diccionario de uso del español. Madrid: Gredos. [DUE] Nisbet, R. (1981). Historia de la idea del progreso. Barcelona: Gedisa.

Porto Dapena, J. (2002). Manual de técnica lexicográfica. Madrid: Arco Libros.

Quiroga Salcedo, C. \& Llull Offenbeck, G. (2008). Léxico del ciclismo. Academia Argentina de Letras: Buenos Aires.

Real Academia Española (1966). Diccionario Histórico de la Lengua Española. Madrid. [DHLE] Real Academia Española (2014). Diccionario de la Lengua Española. Madrid: Planeta. 23a Ed. $<$ http://dle.rae.es> [DLE]

Rodríguez Ortiz, F. (2003). Los ejemplos de las técnicas decimonónicas: el ferrocarril. Asclepio,

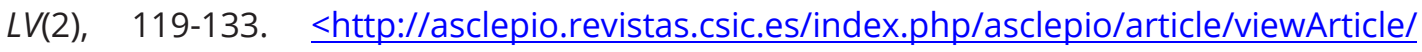
$106>[5 / 4 / 2017]$.

Seco, M. (Dir.) (1999) Diccionario del español actual. Madrid: Santillana. [DEA]

Stubelius, S. (1958). Airship, aeroplane, aircraft. Studies in the history of terms for aircraft in English. Göteborg: Almqvist y Wiksell.

(1960). Balloon, flying-machine, helicopter. Further studies in the history of terms for aircraft in English. Göteborg: Lund.

Viljanen, K. (2007). Wing, aile, Flügel. The origins and development of central aeronautical terms in some languages, Turku.

Vivanco Cervero, V. (1999). Léxico técnico aeronáutico: formación, contaminación y solución. Barcelona: UNED, tesis doctoral.

(2006). El español de la ciencia y la tecnología. Madrid: Arco/Libros. 